\title{
CUSUM control charts with Variable Sampling Interval for monitoring the ratio of two normal variables
}

\author{
Huu Du Nguyen ${ }^{1}$, Kim Phuc Tran*2, and Cédric Heuchenne ${ }^{3}$ \\ ${ }^{1}$ Division of Artificial Intelligence, Dong A University, Danang, \\ Vietnam \\ ${ }^{2}$ Ecole Nationale Supérieure des Arts et Industries Textiles, \\ GEMTEX Laboratory, BP 3032959056 Roubaix Cedex 1, France, \\ *Corresponding author. Email: kim-phuc.tran@ensait.fr \\ ${ }^{3}$ HEC Management School, University of Liège, Liège 4000, Belgium
}

April 1, 2020

\begin{abstract}
In many industrial manufacturing processes, the ratio of two normal random variables plays a key role in ensuring product quality. Monitoring this ratio then becomes an important task that is well worth considering. In this paper, we combine variable sampling (VSI) feature with the cumulative sum (CUSUM) scheme to create new type of control chart for purpose of tracking the ratio of two normal variables. The average time to sign $(A T S)$ is used as a measure to evaluate the performance of VSI CUSUM charts. The sensitive of the charts for different deterministics process shifts as well as random process shifts is displayed in several tables. The results show that our proposed control charts have much more attractive power in comparison with the standard CUSUM charts.
\end{abstract}

Keyword CUSUM, VSI, Ratio distribution, Markov Chain, Statistical Process Control.

\section{Introduction}

As discussed in Celano and Castagliola ${ }^{1}$, designing a control chart for monitoring the ratio $Z$ of two random variables $X$ and $Y$, i.e. $Z=\frac{X}{Y}$, is not straightforward. This is because the distribution of $Z$ has no moments, leading to being impossible to determine its mean and its standard deviation. In addition, it is quite complicated to express the mathematical 
form of probability density function (p.d.f) of $Z$ while an exact expression for its cumulative distribution function (c.d.f) is impossible to obtain Geary $^{2}$; Marsaglia ${ }^{3}$; Hinkley ${ }^{4}$. However, many processes require monitoring the ratios of two (or more) differently random variables in practice. Celano and Castagliola ${ }^{1}$ even pointed out a number of situations where the ratio of two quantities should be used, involving (1) when one concerns the correct proportion of two components in a composition; and (2) when a product quality characteristic is measured as the ratio before and after particular operations like a chemical or physical reaction. Many specific examples of the need to track the ratio of two random variables can be found in Celano and Castagliola ${ }^{1}$. In statistical process control (SPC) literature, Celano et al. ${ }^{6}$ firstly discussed the statistical properties of a Shewhart chart (Shewhart-RZ) for individual measurements and then extended their studies to subgroups consisting of more than 1 sample units in Celano and Castagliola $^{1}$. They also developed the Synthetic-RZ control chart that is more sensitive with small or moderate ratio shifts than Shewhart-RZ chart Celano and Castagliola ${ }^{5}$. Tran et al. ${ }^{7}$ and Tran et al. ${ }^{8},{ }^{9}$ followed theCelano and Castagliola ${ }^{5}$ 's paper by exploring the Run Rules-RZ type chart, the CUSUM-RZ type chart and the EWMA-RZ type chart, respectively. Recently, the control charts designed based on steady-state ARL (average run length) analysis of ARL-unbiased EWMA-RZ for monitoring the ratio of two normal variables are introduced in Tran and Knoth ${ }^{10}$.

One common feature in previously proposed control charts can be realized that the sampling interval is supposed to be fixed from time to time. That is to say, the time interval between two consecutive samples does not change no matter where the first sample is in the control limit. This provokes the adaptive strategies to improve the efficiency of those control charts by observing the dropping point of present sample before deciding on the next sampling interval. It is called the variable sampling interval (VSI) type chart that allows the sampling interval to vary at different levels. If a point represented a sample falls into the control limits but far away from central, it is reasonable to think that the next point will most likely fall outside the limits. The next sampling interval should be shorter to quickly detect the process shifts if they actually exits. On the contrary, if the sample point is close to the target or central limit, it indicates no trouble and one does not need to rush to get the next sample. In many situations, VSI type chart is proven to be more sensitive than those fixed sampling interval (FSI) of the same type chart.

We suggest in this paper integrating the VSI feature into CUSUM control chart on monitoring the ratio between two normal variables. By incorporating these two schemes, it is expected to obtain new type of control chart, say VSI CUSUM-RZ, with better performance in detecting the ratio shifts. 
This new approach can be considered as an extension of the CUSUM-RZ chart based on FSI scheme presented in Tran et al. ${ }^{8}$. We evaluate the performance of the proposed chart via the average time to signal (ATS) and the expected average time to signal (EATS). A new coefficient of control charts are introduced to make the designed charts more realistic.

The rest of the paper is structured as follows. In Section 2, we present the formulae of control limits, the new warning limit coefficient and the implementation of the two one-sided VSI CUMSUM-RZ control charts. Section 3 is devoted to designing the VSI CUSUM-RZ control charts monitoring the ratio of two normal variables. Section 4 shows numerical results and chart performance with a direct comparison between FSI CUSUM-RZ charts and VSI CUSUM-RZ charts. An illustrative example from the real industrial data is provided in Section 5. Some remarks and suggestions are given to conclude in Section 6.

\section{Implementation of VSI CUSUM-RZ control charts}

Let $\mathbf{W}=(X, Y)^{T}$ be a bivariate normal random vector with mean vector $\boldsymbol{\mu}_{\boldsymbol{W}}$ and variance-covariance matrix $\boldsymbol{\Sigma}_{\boldsymbol{W}}$, i.e. $\mathbf{W} \sim N\left(\boldsymbol{\mu}_{\boldsymbol{W}}, \boldsymbol{\Sigma}_{\boldsymbol{W}}\right)$. Consider the ratio of $X$ to $Y, Z=\frac{X}{Y}$. Since both $X$ and $Y$ follows normal distribution, $Z$ represents the ratio of two normal variables. In order to monitor $Z$, a sample of $n$ independent $\left\{\mathbf{W}_{i, 1}, \mathbf{W}_{i, 2}, \ldots, \mathbf{W}_{i, n}\right\}$ at each sampling period $i=1,2, \ldots$ is collected. Each $\mathbf{W}_{i, j}$ is supposed to be a bivariate normal random vector, $\mathbf{W}_{i, j}=\left(X_{i, j}, Y_{i, j}\right)^{T} \sim N\left(\boldsymbol{\mu}_{\mathbf{W}, i}, \boldsymbol{\Sigma}_{\mathbf{W}, i}\right), j=1, \ldots, n$, with mean vector and variance-covariance matrix

$$
\boldsymbol{\mu}_{\mathbf{W}, i}=\left(\begin{array}{c}
\mu_{X, i} \\
\mu_{Y, i}
\end{array}\right) \text { and } \boldsymbol{\Sigma}_{\mathbf{W}, i}=\left(\begin{array}{cc}
\sigma_{X, i}^{2} & \rho \sigma_{X, i} \sigma_{Y, i} \\
\rho \sigma_{X, i} \sigma_{Y, i} & \sigma_{Y, i}^{2}
\end{array}\right),
$$

where $\rho$ is in-control coefficient of correlation between $X$ and $Y$.

We also use the following assumptions as discussed in Celano and Castagliola ${ }^{1}$.

- The sample units are free to change from one subgroup to another, namely the constraints $\boldsymbol{\mu}_{\mathbf{W}, i}=\boldsymbol{\mu}_{\mathbf{W}, k}$ and $\boldsymbol{\Sigma}_{\mathbf{W}, i}=\boldsymbol{\Sigma}_{\mathbf{W}, k}$ are not required for $i \neq k$.

- The coefficients of variations $\gamma_{X}$ and $\gamma_{Y}$ of both variables $X$ and $Y$ are known and constant from sample to sample. That means for every $i$ we have $\sigma_{X, i}=\gamma_{X} \times \mu_{X, i}$ and $\sigma_{Y, i}=\gamma_{Y} \times \mu_{Y, i}$.

- The ratio $\frac{\mu_{X, i}}{\mu_{Y, i}}$ does not depend on the sample as long as the process runs in control; i.e. $\frac{\mu_{X, i}}{\mu_{Y, i}}=z_{0} \forall i=1,2, \ldots$, where $z_{0}$ is a known in-control value for the ratio. 
Under these assumptions, we suggest to monitor the following statistic for the two one-sided VSI CUSUM control charts:

$$
\hat{Z}_{i}=\frac{\hat{\mu}_{X, i}}{\hat{\mu}_{Y, i}}=\frac{\bar{X}_{i}}{\bar{Y}_{i}}=\frac{\sum_{j=1}^{n} X_{i, j}}{\sum_{j=1}^{n} Y_{i, j}}, i=1,2, \ldots
$$

As discussed in Tran et al. ${ }^{8}$, the distribution of $Z$ is skewed and nonsymmetric. Thus, it is preferable to design two separated one-sided CUSUM control charts rather than one two-sided CUSUM control chart. In the case one would like to explore the two-sided chart, the unbiased-ARL measurement should be considered. If not, the performance of the chart corresponding to either decreasing or increasing shifts is very poor, even worse than that the Shewhart chart as discussed in Castagliola et al. ${ }^{11}$. The two one-sided VSI CUSUM-RZ control chart are defined as follows.

- An upward VSI CUSUM-RZ chart aiming at detecting an increase in the ratio that monitors the statistic

$$
S_{i}^{+}=\max \left(0, S_{i-1}^{+}+\left(\hat{Z}_{i}-z_{0}\right)-K^{+}\right),
$$

with the initial value $S_{0}^{+}=0$, the corresponding upper control limit $U C L^{+}=H_{U} \times z_{0}$ and upper warning limit $U W L^{+}=R_{U} \times U C L^{+}$, where $H_{U}>0, R_{U} \in[0,1]$.

- A downward CUSUM chart for detecting a decrease in the ratio that monitors the statistic

$$
S_{i}^{-}=\max \left(0, S_{i-1}^{-}-\left(\hat{Z}_{i}-z_{0}\right)-K^{-}\right),
$$

with the initial value $S_{0}^{-}=0$, the corresponding lower control limit $L C L^{-}=H_{D} \times z_{0}$ and lower warning limit where $L W L^{-}=R_{D} \times$ $L C L^{-}$, where $H_{D}>0, R_{D} \in[0,1]$.

The reference parameters for purpose of tuning the sensitivity of the charts, $K^{+}$and $K^{-}$, are written in the form $K^{+}=z_{0} \times K_{U}$ and $K^{-}=z_{0} \times$ $K_{D}$, where $z_{0}$ is the in-control ratio. The parameters $\left(H_{U}, K_{U}\right)$ or $\left(H_{D}, K_{D}\right)$ is the control chart parameters. The warning limit $U W L^{+}\left(L W L^{-}\right)$is used to determine the proportion that the control statistic falls within the long and short sampling regions, respectively. They are associated with control limit through the warning limit coefficient $R_{U}\left(R_{D}\right)$. The closer to 0 is the warning limit coefficient $R_{U}\left(R_{D}\right)$, the larger is warning region compared to control interval.

The proposed VSI CUSUM-RZ charts works as follows.

i) If the control statistic $S_{i}^{+}\left(S_{i}^{-}\right)$falls within the central region, namely the interval $\left[z_{0}, U W L^{+}\right]$or $\left(\left[L W L^{-}, z_{0}\right]\right)$, there is no reason to say that the process have been shifted: it is believed to be still in safety. Therefore, the next longer sampling interval, say $h_{L}$, is applied. 


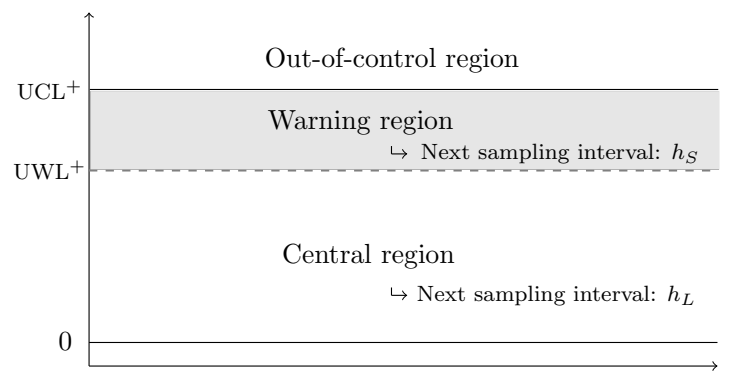

Figure 1: Three regions and operation rules of the Upward VSI EWMA-RZ charts

ii) If the control statistic $S_{i}^{+}\left(S_{i}^{-}\right)$drops into warning region, i.e. the interval $\left[U W L^{+}, U C L^{+}\right]$or $\left(\left[L C L^{-}, L W L^{-}\right]\right)$, it is reasonable to suspect that the process seems to be put at risk. As a result, the next sampling interval should be shorter, say $h_{S}$, to quickly detect process shifts if they occur.

iii) An alarming signal is given at time $i$ if $S_{i}^{+}$or $S_{i}^{-}$is beyond the control limit $\left(S_{i}^{+}>U C L^{+}\right.$or $\left.S_{i}^{-}<L C L^{-}\right)$; the process is signaled to be out-of-control.

Figure 1 illustrates a graphical view of the operation of the upward VSI CUSUM-RZ chart.

\section{Design of optimal VSI CUSUM-RZ control charts}

We discuss in this Section a method to measure the performance of proposed two one-sided VSI CUSUM-RZ control charts. In practice, there are two commonly used measures to evaluate the control chart effectiveness, involving average run length $(A R L)$ and average time to signal $(A T S)$. While $A R L$ describes the average number of sample before a control chart signals an out-of-control conditions or issues a false alarm, the ATS represents the corresponding expected time to signal. Due to varying sampling interval, the $A R L$ is not really an appropriate measure in the VSI type charts. We therefore assess the power of our chart by using the measure ATS. It is denoted by $A T S_{0}$ or $A T S_{1}$ corresponding to the in-control or out-of-control value of the $A T S$.

Suppose that when an assignable cause occurs, the in-control ratio $z_{0}$ is shifted to $z_{1}=\tau \times z_{0}$ and the coefficient of correlation is shifted from $\rho=\rho_{0}$ to $\rho=\rho_{1}$, where $\tau>0$ is the shift size. For the defined value of $z_{0}$, the performance of the chart dose not depend on $z_{0}$. Without loss of generality, 


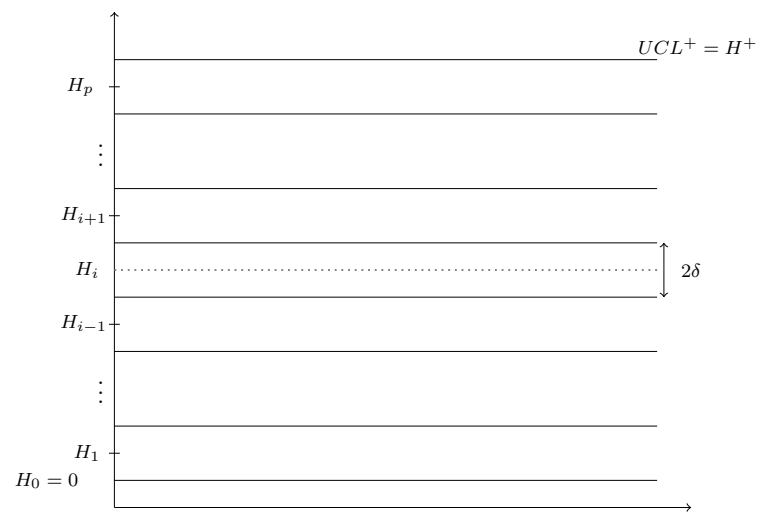

Figure 2: Three regions and operation rules of the Upward VSI CUSUM-RZ charts

we can assume that $z_{0}=1$. We will use a Markov chain approach to calculate the ATS. Firstly, the control interval is partitioned $p$ sub-intervals of width $2 \delta$ where $\delta=\frac{U C L^{+}}{2 p}\left(\delta=\frac{L C L^{-}}{2 p}\right)$ corresponding to $p+2$ states of the Markov chain as demonstrated in Figure 2. In this Figure, the first line corresponds to the state 0 ; each other sub-intervals, say $\left(H_{j}-\delta, H_{j}+\delta\right]$, represents the in-control transient state $j$ of Markov chain, $j=1, . ., p$ while the state $p+2$ is out-of-control or absorbing state one corresponds to a signal from control chart. If the statistic $S_{i}^{+}\left(S_{i}^{-}\right)$drops into the sub-interval $j$, the Markov chain is in the transient sate $j$ for sample $i$; if not, the chain reaches absorbing state. The number of sub-intervals $p$ is chosen sufficiently large (say $p=200$ in this paper) so that each $H_{j}, j=1, \ldots, p$ can be considered as the midpoint of the $j^{\text {th }}$ sub-interval. This also makes sure that the method is effective, allowing the time to signal properties $(A T S)$ to be accurately evaluated. The transition probability matrix $\mathbf{P}$ of the discrete Markov chain is

$$
\mathbf{P}=\left(\begin{array}{cc}
\mathbf{Q} & \mathbf{r} \\
\mathbf{0}^{T} & 1
\end{array}\right)=\left(\begin{array}{ccccc}
Q_{0,0} & Q_{0,1} & \ldots & Q_{0, p} & r_{0} \\
Q_{1,0} & Q_{1,1} & \ldots & Q_{1, p} & r_{1} \\
\vdots & \vdots & & & \vdots \\
Q_{p, 0} & Q_{p, 1} & \ldots & Q_{p, p} & r_{p} \\
0 & 0 & \ldots & 0 & 1
\end{array}\right)
$$

where $\mathbf{Q}$ is the $(p+1, p+1)$ matrix of transient probabilities, $\mathbf{0}=(0,0, \ldots, 0)^{T}$ and the $(p+1)$ vector $\mathbf{r}$ satisfies $\mathbf{r}=(\mathbf{1}-\mathbf{Q} \mathbf{1})$ (row probabilities must sum to 1$)$ with $\mathbf{1}=(1,1, \ldots, 1)^{T}$. Denote $\mathbf{q}$ the $(p+1,1)$ vector of initial probabilities associated with the $p+1$ transient states. In particular, $\mathbf{q}=(1,0, \ldots, 0)^{T}$ is corresponding to the "restart state". A representative element $Q_{i, j}, i=0,1, \ldots, p$, of the matrix $\mathbf{Q}$ is calculated by 
- for the upward chart,

$$
\begin{aligned}
\text { if } j=0: & Q_{i, 0}=F_{Z}\left(1-H_{i}+k^{+} \mid \frac{\gamma_{X}}{\sqrt{n}}, \frac{\gamma_{Y}}{\sqrt{n}}, \frac{\tau \gamma_{X}}{\gamma_{Y}}, \rho_{1}\right) ; \\
\text { if } j=1,2, \ldots, p: & \\
Q_{i, j}=F_{Z} & \left(1+H_{j}-H_{i}+\delta+k^{+} \mid \frac{\gamma_{X}}{\sqrt{n}}, \frac{\gamma_{Y}}{\sqrt{n}}, \frac{\tau \gamma_{X}}{\gamma_{Y}}, \rho_{1}\right) \\
& \quad-F_{Z}\left(1+H_{j}-H_{i}-\delta+k^{+} \mid \frac{\gamma_{X}}{\sqrt{n}}, \frac{\gamma_{Y}}{\sqrt{n}}, \frac{\tau \gamma_{X}}{\gamma_{Y}}, \rho_{1}\right),
\end{aligned}
$$

- For the downward chart,

$$
\begin{gathered}
\text { if } j=0: \quad Q_{i, 0}=1-F_{Z}\left(1+H_{i}-k^{-} \mid \frac{\gamma_{X}}{\sqrt{n}}, \frac{\gamma_{Y}}{\sqrt{n}}, \frac{\tau \gamma_{X}}{\gamma_{Y}} ; \rho_{1}\right), \\
\text { if } j=1,2, \ldots, p: \\
\begin{aligned}
Q_{i, j}=F_{Z} & \left(1+H_{j}-H_{i}+\delta+k^{+} \mid \frac{\gamma_{X}}{\sqrt{n}}, \frac{\gamma_{Y}}{\sqrt{n}}, \frac{\tau \gamma_{X}}{\gamma_{Y}}, \rho_{1}\right) \\
& -F_{Z}\left(1+H_{j}-H_{i}-\delta+k^{+} \mid \frac{\gamma_{X}}{\sqrt{n}}, \frac{\gamma_{Y}}{\sqrt{n}}, \frac{\tau \gamma_{X}}{\gamma_{Y}}, \rho_{1}\right) .
\end{aligned}
\end{gathered}
$$

The function $F_{Z}($.$) in equations (5) to (8) is the cumulative distribution$ function (c.d.f) of $Z$ defined by

$$
F_{Z}\left(z \mid \gamma_{X}, \gamma_{Y}, \omega, \rho\right) \simeq \Phi\left(\frac{A}{B}\right),
$$

where $\Phi($.$) is the c.d.f. of the standard normal distribution; A$ and $B$ are functions of $z, \gamma_{X}, \gamma_{Y}, \omega$ and $\rho$, i.e.

$$
\begin{aligned}
A & =\frac{z}{\gamma_{Y}}-\frac{\omega}{\gamma_{X}}, \\
B & =\sqrt{\omega^{2}-2 \rho \omega z+z^{2}} .
\end{aligned}
$$

This approximation of $F_{Z}($.$) in (9) and also an approximation of the$ density function as well as the inverse cumulative distribution function of $Z$ are avaiable in a number of previous papers monitoring the ratio, for example, see Celano and Castagliola ${ }^{1}$, Tran et al. ${ }^{7}$.

From previous definitions, ATS can be computed by following the formulae as in Saccucci and J.M. ${ }^{12}$ :

$$
A T S=\mathbf{q}^{T}(\mathbf{I}-\mathbf{Q})^{-1} \mathbf{g},
$$

where $\mathbf{I}$ is the identity matrix, $\mathbf{g}$ is the vector of sampling intervals corresponding to the discrete states o the Markov chain. The $j^{\text {th }}$ element $g_{j}$ 
of the vector $\mathbf{g}$ is the sampling interval when the control statistic is in the state $j$ (denoted by $H_{j}$ ). That means $g_{j}=h_{L}$ if $H_{j}$ is in central region and $g_{j}=h_{S}$ if $H_{j}$ is in warning region.

In FSI model, the sampling interval is fixed, the $A T S$ is then simply a multiple of the $A R L$, i.e. $A T S^{\mathrm{FSI}}=h_{F} \times A R L^{\mathrm{FSI}}$, where $h_{F}$ stands for the constant time between two successive samples. For a VSI model, the $A T S$ depends on the sampling interval. That is $A T S^{\mathrm{VSI}}=E(h) \times A R L^{\mathrm{VSI}}$, where $E(h)$ is the average sampling interval. From the computation of $A R L$ in Brook and Evans ${ }^{13}, E(h)$ is calculated by

$$
E(h)=\frac{\mathbf{q}^{T}(\mathbf{I}-\mathbf{Q})^{-1} \mathbf{g}}{\mathbf{q}^{T}(\mathbf{I}-\mathbf{Q})^{-1} \mathbf{1}} .
$$

In order to make sure of a fair comparison with FSI charts, it is necessary to constrains both $A T S_{0}$ and $E_{0}(h)$ (the in-control average sampling interval). Their values should be the same as those in FSI-type control charts as discussed in Castagliola ${ }^{14}$. We assume that the in-control expected sampling interval of the VSI chart is set equal to $E_{0}(h)=1$ time unit, as normal in FSI chart. As a result, we obtain $A T S_{0}=A R L_{0}$ for both FSI and VSI type control charts.

In procedure of designing VSI type chart, a fixed couple $\left(h_{S}, h_{L}\right)$ from a list is ussually used as in W.C.Yeong ${ }^{15}$ and Castagliola et al. ${ }^{11}$. However, we suppose that predetermining the value of $h_{L}$ is not really convincing. When the sample point falls into safe region, it indicates no trouble. The sampling interval should be chosen to optimize the performance of the chart rather than being fixed before. The value of $h_{S}$ is different, it should not be free to choose because it relates to the time of product manufacture. We then propose to consider $h_{L}$ as the optimal parameter and predetermine the values of $R_{U}\left(R_{D}\right)$ and $h_{S}$. Thus, we optimize the chart performance by the optimal parameters $\left(K_{U}, H_{U}, h_{L}\right)$ (upward chart) and $\left(K_{D}, H_{D}, h_{L}\right)$ (downward chart), using small but non-zero values of $h_{S}$. Our suggested algorithm is different with those in previous works performed by fixing both $h_{S}$ and $h_{L}$. That is to say, for specific value of shift size, we look for the triple $\left(K_{U}^{*}, H_{U}^{*}, h_{L}^{*}\right)$ or $\left(K_{D}^{*}, H_{D}^{*}, h_{L}^{*}\right)$ satisfying

- for downward chart,

$$
\left(K_{U}^{*}, H_{U}^{*}, h_{L}^{*}\right)=\underset{\left(K_{U}, H_{U}, h_{L}\right)}{\operatorname{argmin}} \operatorname{ATS}\left(n, K_{U}, H_{U}, R_{U}, \rho, \gamma_{X}, \gamma_{Y}, \tau, h_{L}, h_{S}\right)
$$

subject to the constraints $A T S_{0}=A T S_{0}$ and $E_{0}(h)=1$, 
- for upward chart,

$$
\left(K_{D}^{*}, H_{D}^{*}, h_{L}^{*}\right)=\underset{\left(K_{D}, H_{D}, h_{L}\right)}{\operatorname{argmin}} \operatorname{ATS}\left(n, K_{D}, H_{D}, R_{D}, \rho, \gamma_{X}, \gamma_{Y}, \tau, h_{L}, h_{S}\right)
$$

subject to the constraints $A T S_{0}=A T S_{0}$ and $E_{0}(h)=1$.

Using ATS as a measure for evaluating the performance of VSI CUSUMRZ control chart requires to predetermine a deterministic shift size $\tau$. This pre-determination, however, is not straightforward for quality practitioners. Castagliola et al. ${ }^{11}$ presented a number of comments about the reason for the difficulty of predicting the exact value of the size of a shift to the outof-control condition. In order to overcome the situation, one can choose to utilize EATS (expected average time to signal) as a replacement for $A T S$ :

$$
E A T S=\int_{\Omega} A T S \times f_{\tau}(\tau) \mathrm{d} \tau
$$

where $\Omega$ is support of shift size $\tau$ and $f_{\tau}(\tau)$ is density function of $\tau$. The convenience of this alternative method is that the practitioners do not need to predefined any specifically unknown shift size. In practice, the quality practitioners might have some preferences about the range of shift sizes that they want the chart to be able to detect. Therefore, the uniform distribution for $\tau$ on fixed support $\Omega=[a, b$,$] is usually proposed, i.e. f_{\tau}(\tau)=\frac{1}{b-a}$ over the possibly guessed interval $[a, b$,$] . Our numerical analysis now include finding$ out the new optimal triples $\left(K_{U}^{*}, H_{U}^{*}, h_{L}^{*}\right)$ or $\left(K_{D}^{*}, H_{D}^{*}, h_{L}^{*}\right)$ which minimize the out-of-control $E A T S_{1}$ subject to the same constraints in equations (14) and (15).

In our study, these optimal combinations above are found by simultaneously using a non-linear equation solver coupled to an optimization algorithm (developed with Scicoslab software).

\section{Numerical results and comparison}

We discover in this section the statistical performance of the two onesided VSI CUMSUM-RZ charts subject to the constraints $A T S_{0}=200$ and $E_{0}(h)=1$. For the sake of brevity, assume that the correlation coefficient between two variables does not depend on the condition of the process, i.e. $\rho_{1}=\rho_{0}$. The numerical results corresponding to the values of $\rho_{1}$ different from $\rho_{0}$ are not presented in this paper but are available upon request from authors. Many situations of parameters are covered as follows.

- $\gamma_{X} \in\{0.01,0.2\}, \gamma_{Y} \in\{0.01,0.2\}$, coressponding to small or large variation in $X$ and $Y$.

- $\rho_{0} \in\{0.0, \pm 0.4, \pm 0.8\}$, describing the degree of correlation between $X$ and $Y$. 
- $n \in\{1,15\}$, denoting the small or large sample sizes.

- $\tau \in\{0.9,0.95,098,0.99\}$, the decrease ratio shifts and $\tau=\{1.01,1.02$, $1.05,1.1\}$, the increase ratio shifts.

- $R_{U}, R_{D} \in\{0.1,0.2,0.3,0.5\}$, coressponding to small, moderate or equal safe regions compared to warning region.

- $h_{S} \in\{0.1,0.5\}$.

Given the values of $\gamma_{X}, \gamma_{Y}, \rho_{0}, n, h_{S}$ and $R$, the optimal triple $\left(K_{U}^{*}, H_{U}^{*}, h_{L}^{*}\right)$ in equation (14) for upward chart and $\left(K_{D}^{*}, H_{D}^{*}, h_{L}^{*}\right)$ in equation (15) for downward chart are solved. The out-of-control $A T S_{1}$ are then calculated from these optimal triples. The obtained results are displayed in Table 1 to 8 for the deterministic shift sizes. These results are also compared directly to those corresponding parameters in FSI CUSUM chart. A number of general conclusions can be drawn from these obtained results as follows.

- In comparison with those the value of $A R L_{1}$ in FSI CUMSUM-RZ charts, the values of $A T S_{1}$ in VSI CUSUM-RZ charts are smaller in most cases. That means the VSI CUSUM-RZ control charts are faster and more sensitive in detecting the assignable causes in most cases, leading to the conclusion that they in general outperform the standard CUSUM charts. For example, with $\gamma_{X}=0.2, \gamma_{Y}=0.01, \rho_{0}=$ $0.4, R_{D}=0.1, h_{S}=0.1, \tau=0.99$ and $n=15$ in Table 8 , we get $A R L_{1}=55.2$ while $A T S_{1}=36.9$.

- The coefficient of variation $(\mathrm{CV})\left(\gamma_{X}, \gamma_{Y}\right)$ of two variables have substantial influence on the performance of the proposed charts. The charts have considerably better power in detecting out-of-control condition with small values of $\gamma_{X}$ and $\gamma_{Y}$. Taking the case that the parameters $h_{S}=0.5, n=15, R_{U}=0.2, \rho=-0.4$ and $\tau=1.02$. as an example; we have $A T S_{1}=1.4$ for $\gamma_{X}=\gamma_{Y}=0.01$ (Table 2) and $A T S_{1}=39.1$ for $\gamma_{X}=\gamma_{Y}=0.2$ (Table 4). Moreover, the values of $\gamma_{X}$ and $\gamma_{Y}$ also affect the control limits and the sampling interval $h_{L}$. In particular, the rise of the CV of both $X$ and $Y$ leads to the increase of $\left(H_{U}^{*}, h_{L}^{*}\right)$ or $\left(H_{D}^{*}, h_{L}^{*}\right)$. For instance, $\left(H_{D}^{*}, h_{L}^{*}\right)=(0.015,1.233)$ (Table 2) when $\gamma_{X}=\gamma_{Y}=0.01$ and $\left(H_{D}^{*}, h_{L}^{*}\right)=(0.256,1.310)$ when $\gamma_{X}=0.01, \gamma_{Y}=0.2$ (Table 6 ) with the same other parameters of $R_{D}=0.3, n=15, h_{S}=0.1, \tau=0.9, \rho=0.0$

- The change of correlation between two variables $\rho$, from small to large and from negative to positive, has a positive influence on the performance of the VSI CUSUM-RZ charts. Besides, it generally narrows the width of the control intervals of the proposed charts. For example, with $n=1, \gamma_{X}=0.01, \gamma_{Y}=0.2, \rho=0.9, R_{D}=0.5$ and 
$h_{S}=0.5$, we obtain $\left(A T S_{1}, H_{U}^{*}\right)=(15.7,0.775)$ for $\rho=-0.4$ while $\left(A T S_{1}, H_{U}^{*}\right)=(14.7,0.701)$ for $\rho=0.4$ (Table 5). However, the trend of the values of the longer sampling interval $h_{L}$ are not identical when the correlation between two variables $X$ and $Y$ varies. It is more dependent on other parameters. By this result, it can be said that correlation $\rho$ is less influence on the trend of $h_{L}$.

- The predetermined value of $h_{S}$ has impact on both the performance of the chart and the longer sampling interval but with different trends. While the small values of $h_{S}$ result in smaller value of $A T S_{1}$ (corresponding to the better charts performance), they make the sampling interval in safe region, $h_{L}$, larger. For example, in Table 7 with $\gamma_{X}=0.2, \gamma_{Y}=0.01, n=1, R_{U}=0.2, \rho=-0.8$ and $\tau=1.02$, we observe $A T S_{1}=75.3$ and $h_{L}^{*}=2.114$ for $h_{S}=0.1$, but $A T S_{1}=83.1$ and $h_{L}^{*}=1.593$ for $h_{S}=0.5$. It is also interesting to see that the values of $h_{S}$ do not affect the control limits $H_{U}$ or $H_{D}$ and the parameter $K_{U}$ or $K_{D}$ of the chart. In every tables, we see that the value of the couple $\left(H_{U}^{*}, K_{U}^{*}\right)$ or $\left(H_{D}^{*}, K_{D}^{*}\right)$ are almost identical from the blocks that $h_{S}=0.1$ to the blocks that $h_{S}=0.5$.

- The performance of the control charts is strongly affected by the sample size $n$. The larger value of $n$ is, the smaller value of $A T S_{1}$ is. For example, $A T S_{1}=82.7$ when $n=1$ (Table 5) and $A T S_{1}=16.2$ when $n=15$ (Table 6) for the same given values $\gamma_{X}=0.01, \gamma_{Y}=0.2, \rho_{0}=$ $-0.4, R_{D}=0.2, h_{S}=0.1$ and $\tau=0.98$. On the other side, the increase of $n$ reduces the control intervals. For instance, with the same values $\gamma_{X}=\gamma_{Y}=0.2, \rho=0.4, R_{D}=0.5, h_{S}=0.1$ and $\tau=0.95$, we have $H_{D}^{*}=1.755$ for $n=1$ while $H_{D}^{*}=0.314$ for $n=15$.

- Given the value of $\gamma_{X}, \gamma_{Y}, n, \rho, \tau$ and $h_{S}$, the value of $A T S_{1}$ and $h_{L}^{*}$ varies with the predefined value of warling limit coefficient. In general, the smaller is the value of $R_{U}\left(R_{D}\right)$ ), the smaller is the value of $A T S_{1}$ and the larger is the value of $h_{L}^{*}$. For example, $\left(h_{L}^{*}, A T S_{1}\right)=$ $(1.961,7.9)$ for $R=0.1$ and $\left(h_{L}^{*}, A T S_{1}\right)=(1.116,11.1)$ for $R_{D}=0.5$ when $\gamma_{X}=\gamma_{Y}=0.01, \rho=-0.4, n=1, h_{S}=0.1$ and $\tau=0.99$ in Table 1. The better power of the control charts corresponding to the smaller of warning limit coefficient can be explained by the fact that the smaller of $R_{U}\left(R_{D}\right)$ leads to larger warning region in comparison with safe region. As a result, the charts are able to detect faster the shift of the process, but they also tend to require faster sampling interval. However, the influence of $R_{U}$ or $R_{D}$ on the charts be effected by the value of $\gamma_{X}, \gamma_{Y}$ and $n$. When $\gamma_{X}, \gamma_{Y}$ are small and $n$ is large, the performance of the VSI CUSUM-RZ is somewhat better with the value of $R_{U}\left(R_{D}\right)$ equal to 0.3 , see Table 2 . 
It is also worth to consider that, among the change of process parameters, the variation of $\gamma_{X}$ and $\gamma_{Y}$ has the most influential. The performance of the charts is very good for the case these coefficient of variation are small, as comparing results displayed in Table 1 and Table 2 with those in other tables.

For the case of unknown shift size, we consider two different ranges $\Omega_{D}=[0.9,1)$ for decreasing case and $\Omega_{I}=(1,1.1]$ for increasing case. The values of the $E A T S_{1}$ and $E A R L_{1}$ (in FSI charts) corresponding to obtained optimal triples are presented in Table 9 - 12. There are some more remarks obtained from these tables as follows.

- In general, an analogous tendency of the effect of chart parameters are observed as for the deterministic shift size discussed above.

- In many situations, the value of $E A T S_{1}$ for both cases are the same, namely the performance of the chart is approximate symmetrical. For example, in Table 9, when $\left(\gamma_{X}, \gamma_{Y}\right)=(0.01,0.01), n=15, \rho=$ $0.4, h_{S}=0.1$, and $R_{U}=R_{D}=0.2$, it results $E A T S_{1}=1.5$ for both the increasing and decreasing cases. In some other situations, especially when $R_{U}\left(R_{D}\right)$ and $h_{S}$ are small, the $E A T S_{1}$ in decreasing case is much smaller than that the $E A T S_{1}$ in increasing case. That means in such situations, the VSI CUSUM-RZ can detect the downward ratio shift faster than the upward ratio shift. For instance, in Table 11 with $h_{S}=0.1, R_{D}=R_{U}=0.1, \rho=0.0$ and $n=1$, we get $E A T S_{1}=39.8$ for decreasing case and $E A T S_{1}=45.4$ for increasing case.

\section{Illustrative example}

We illustrate in this section an example of the implementation of the VSI CUSUM-RZ control chart to monitor the ratio of two normal variables for the food industry. The dataset is based on that used by Celano and Castagliola ${ }^{1}$. A muesli brand recipe is produced by a food company using a mixture of several ingredients such as sunflower oil, wildflower honey, some kind of seeds like flaxseeds and pumkin, coconut milk powder, rolled oats. The quailty of the recipe require equal weights of "pumpkin seeds" and "flaxseeds". Their nominal proportions to the total weight of box content are both fixed at $p_{p}=p_{f}=0.1$. Let $\mu_{p, i}$ and $\mu_{f, i}$ denote the mean weights of "pumpkin seeds" and "flaxseeds". Due to different dimensions of boxes, the quality practitioner would perform on-line SPC monitoring at regular intervals $i=1,2, \ldots$ to check deviations from the in-control ratio $z_{0}=\frac{\mu_{p, i}}{\mu_{f, i}}=1$. In the case of problems occurring at the dosing machine during the mixing process, the in-control ratio can experience undesired shifts. The quality practitioner decides to sample $n=5$ boxes every 30 


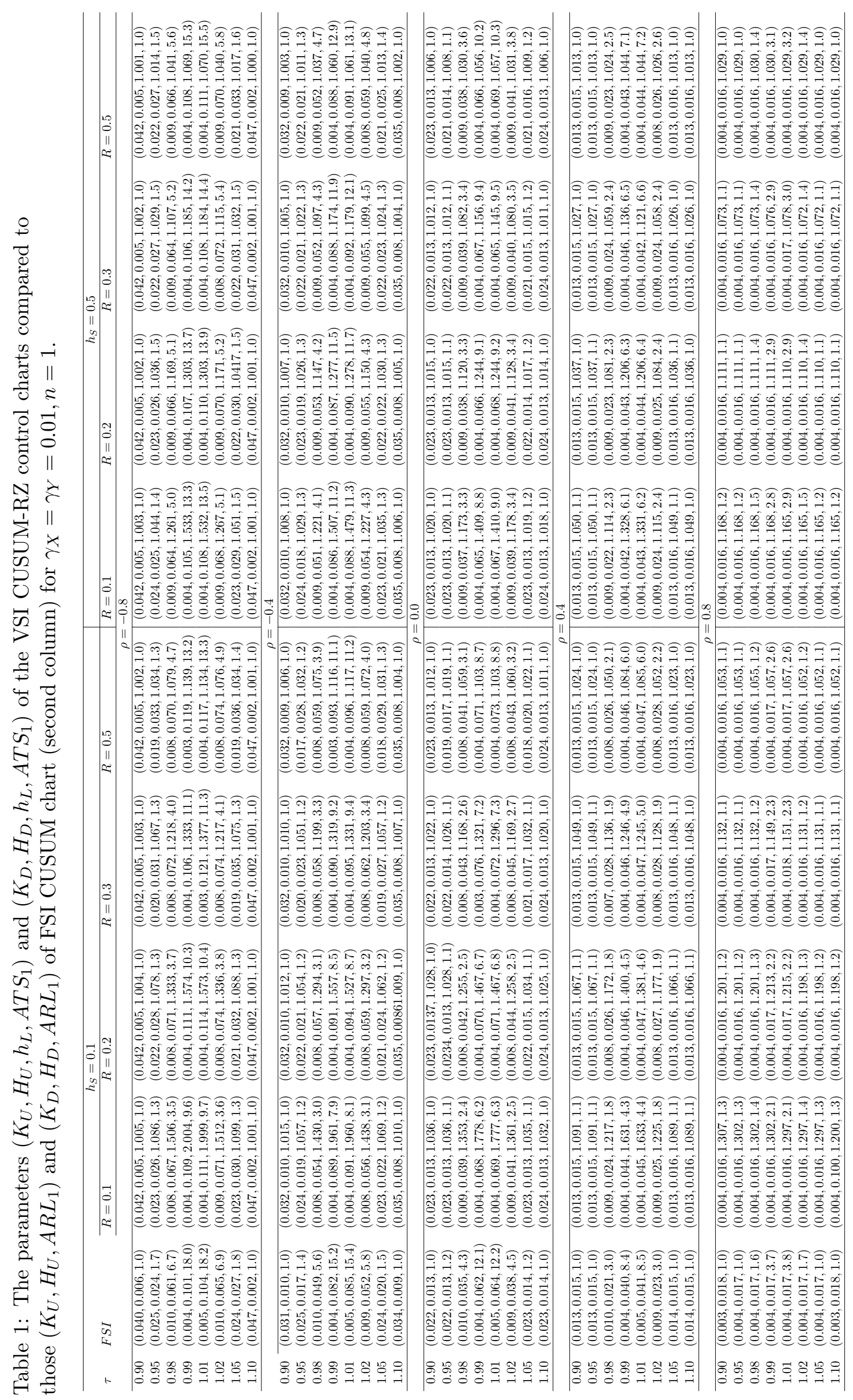




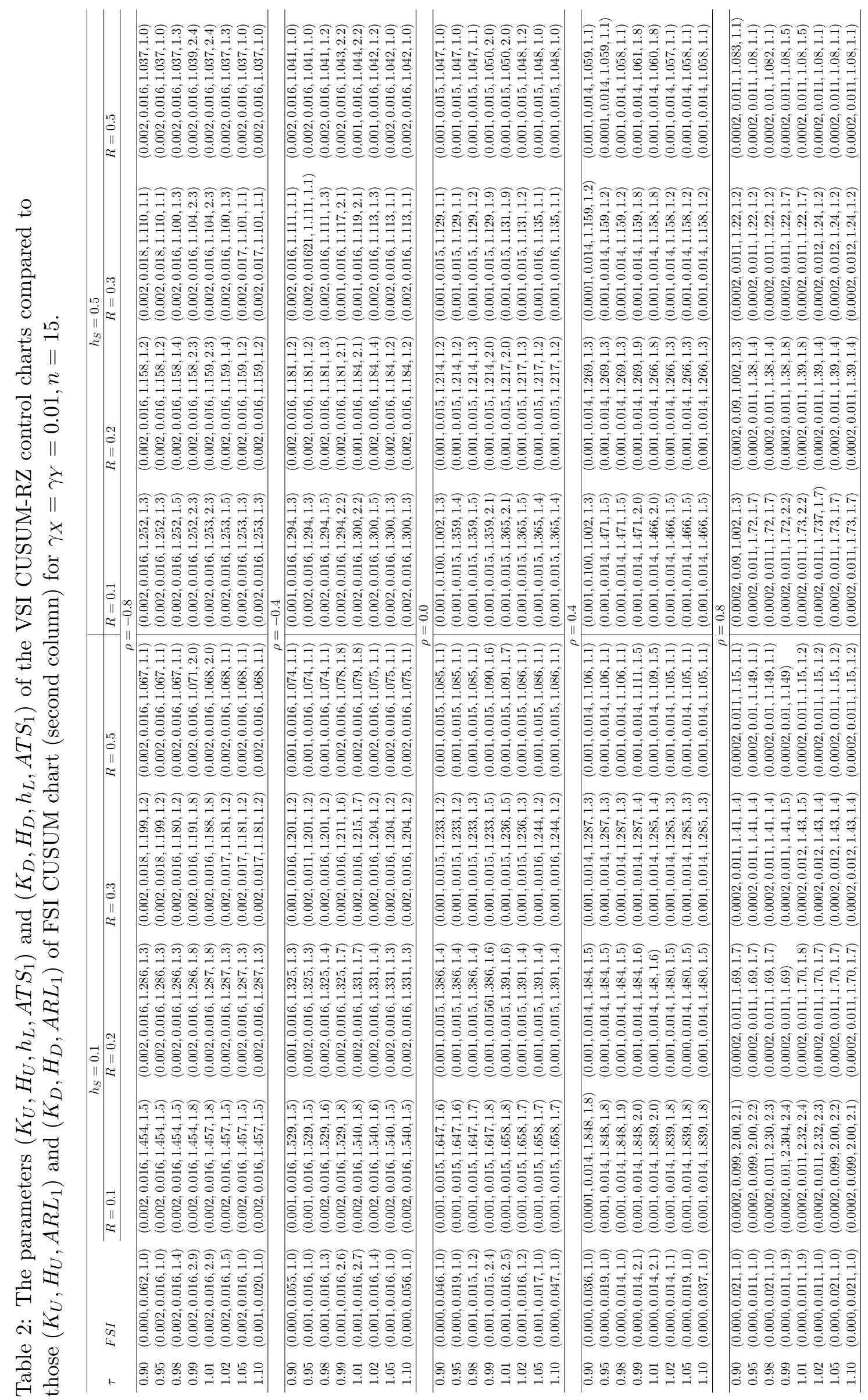




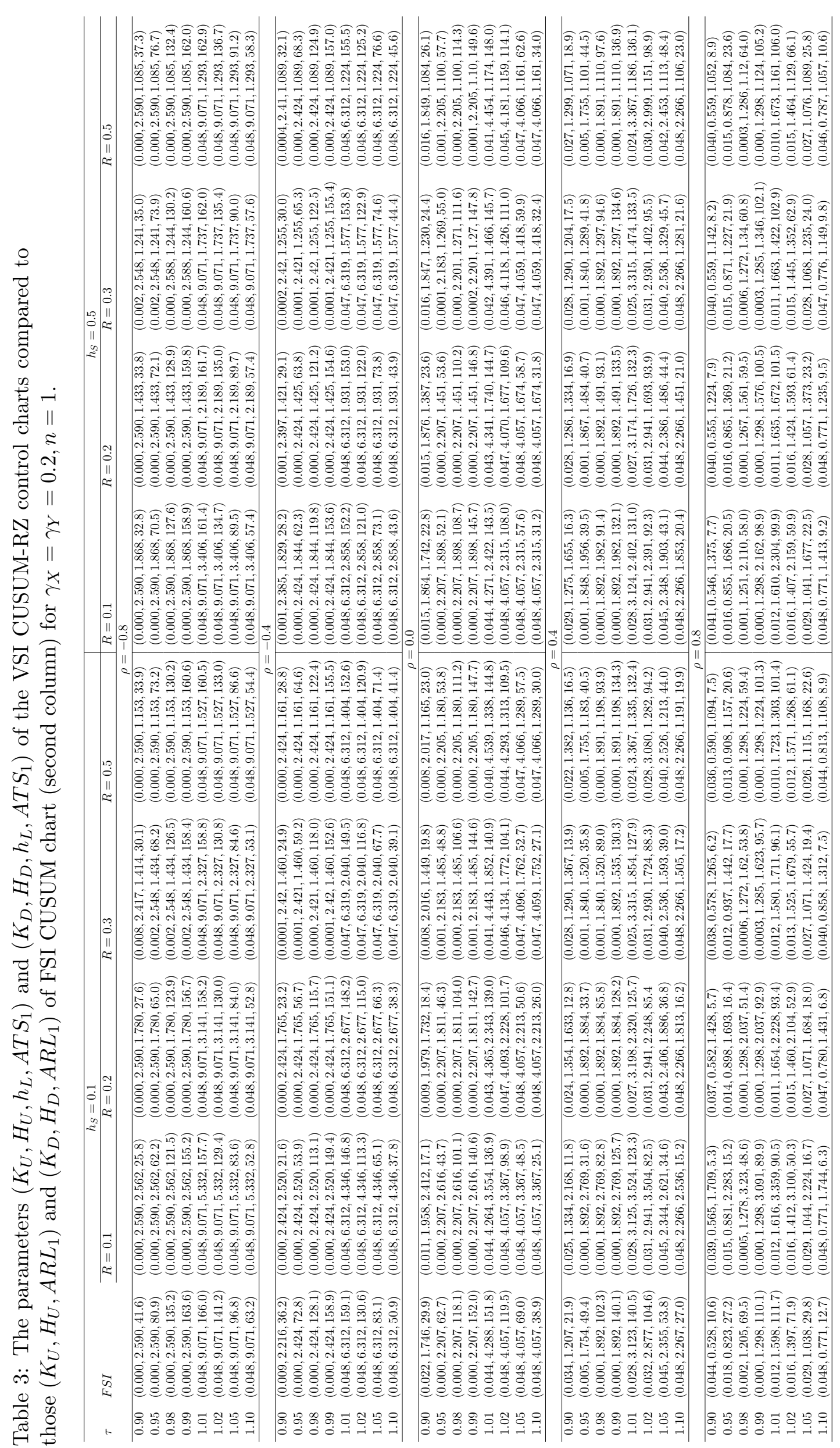




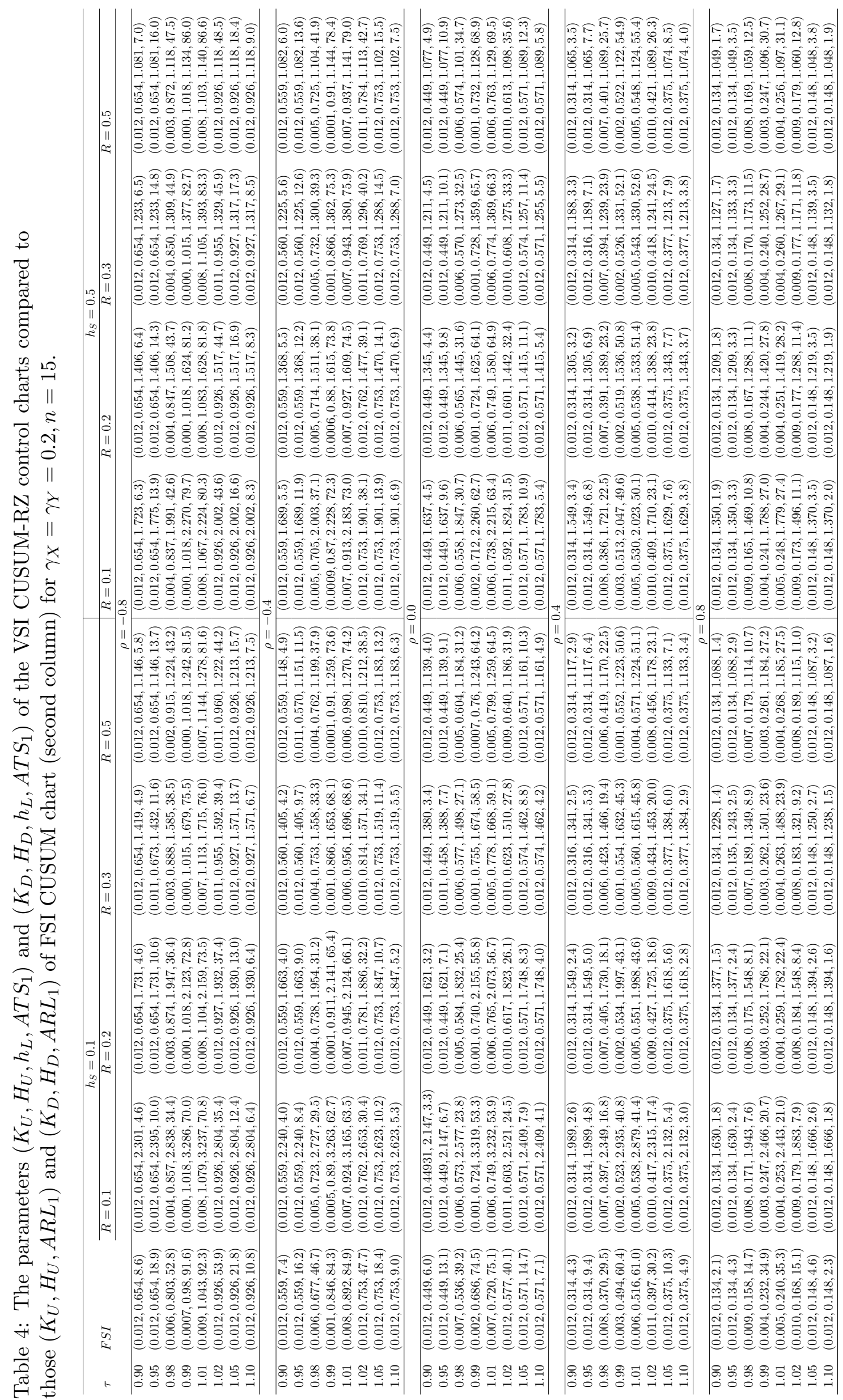




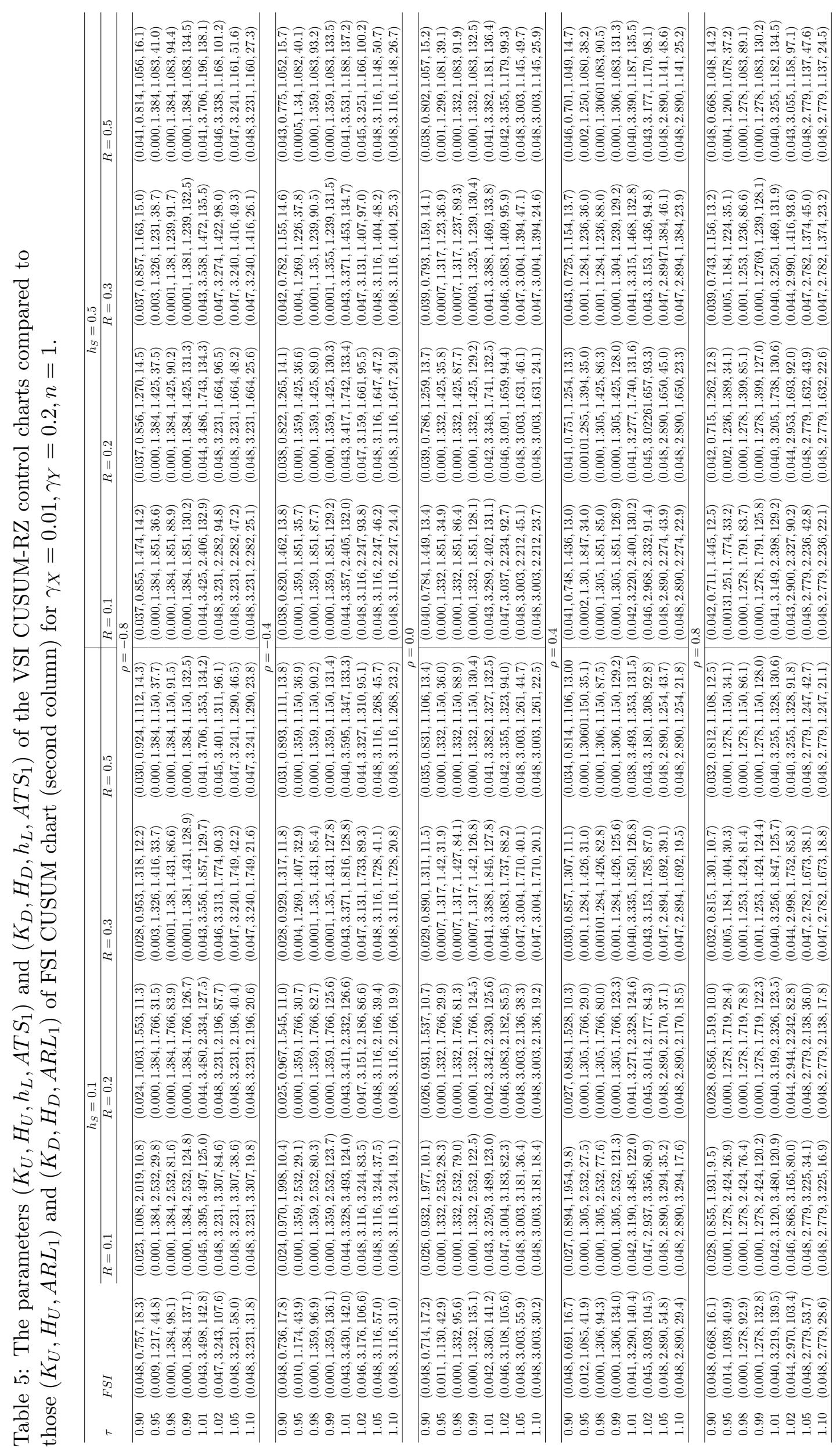




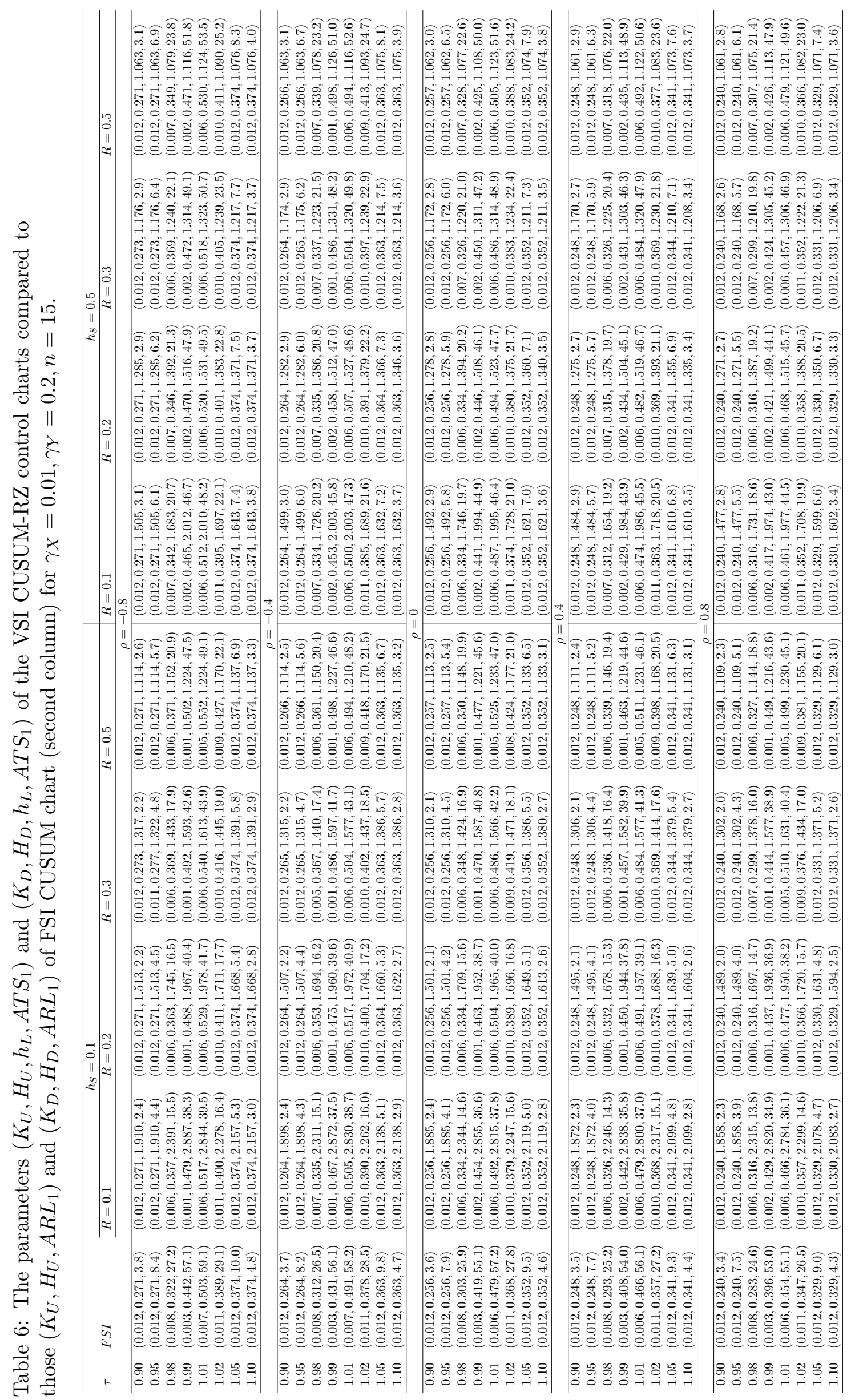




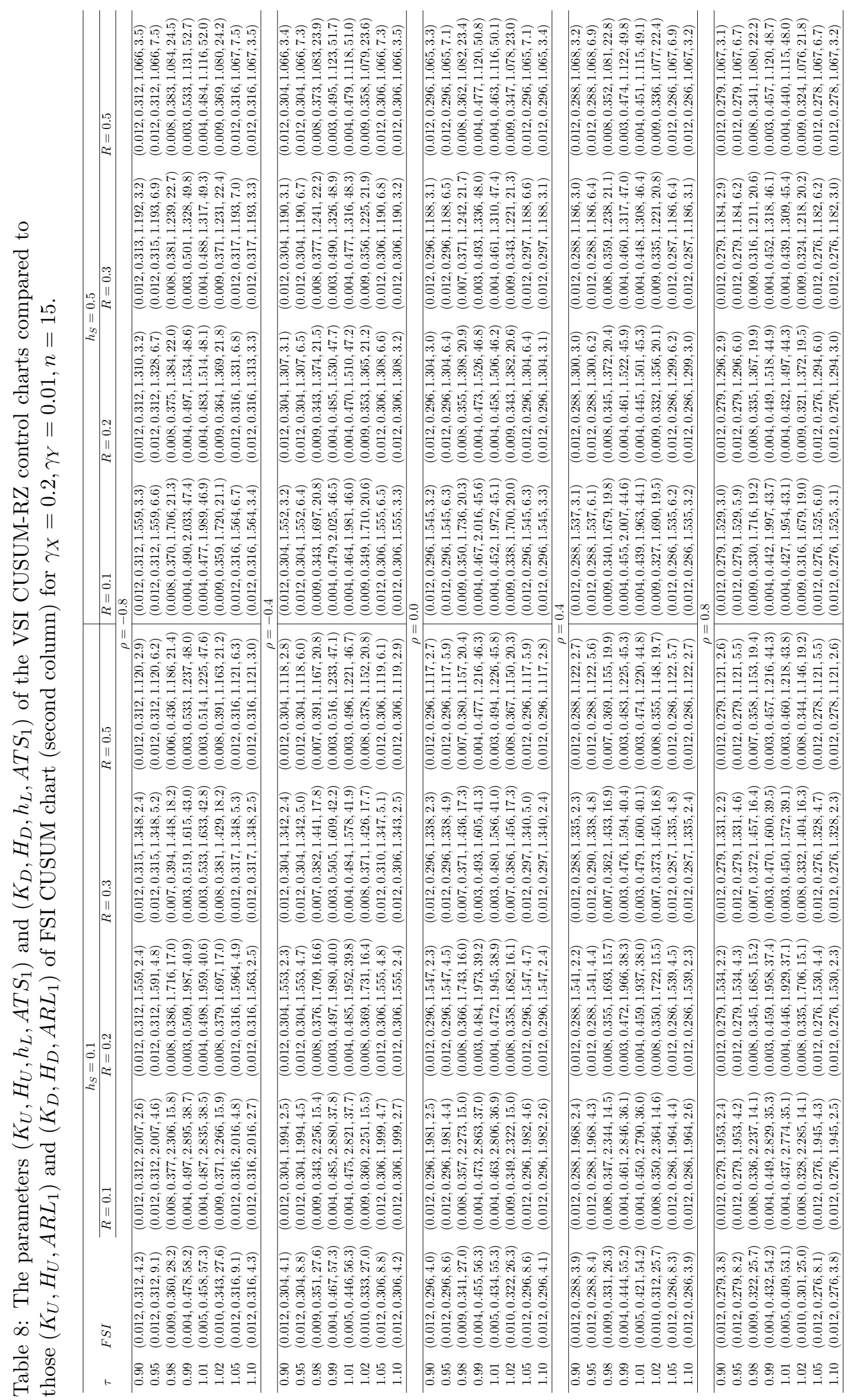




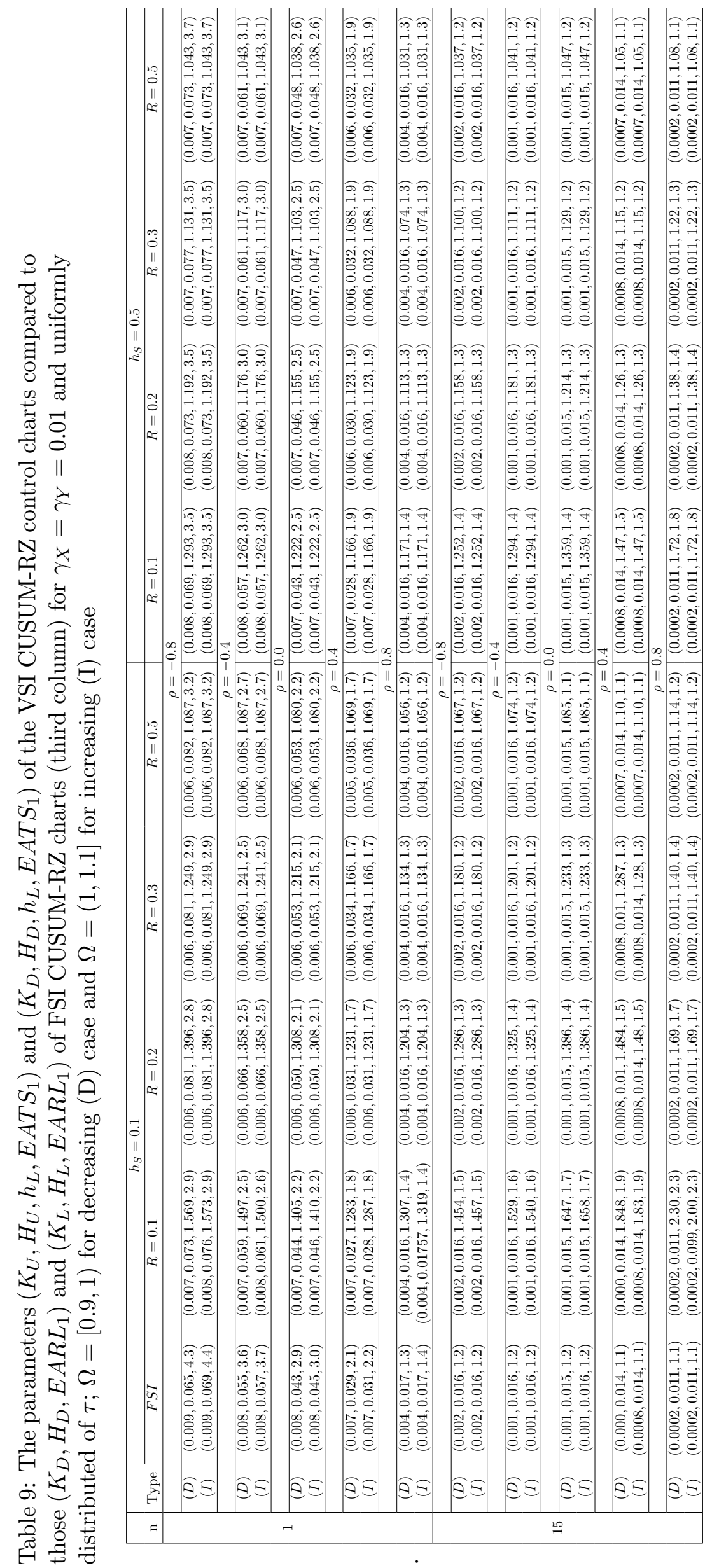




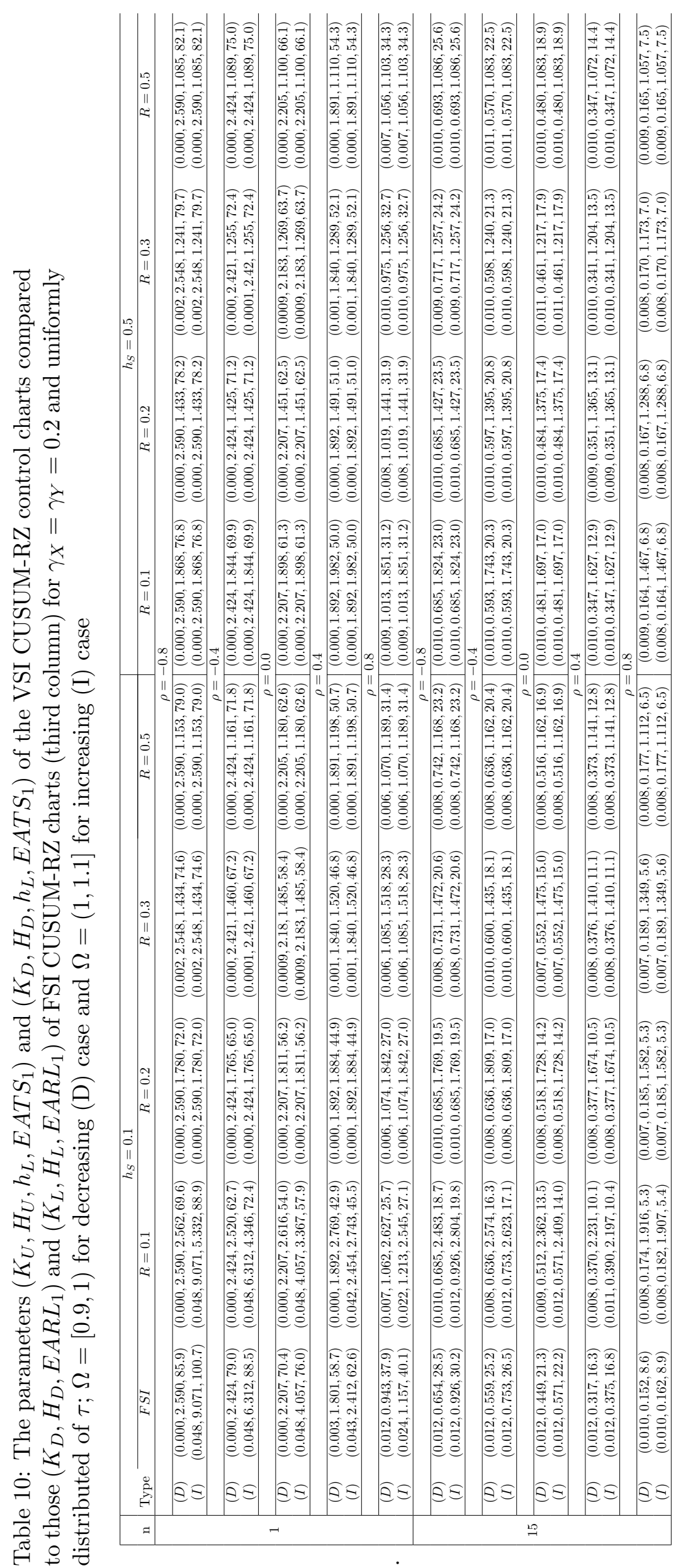




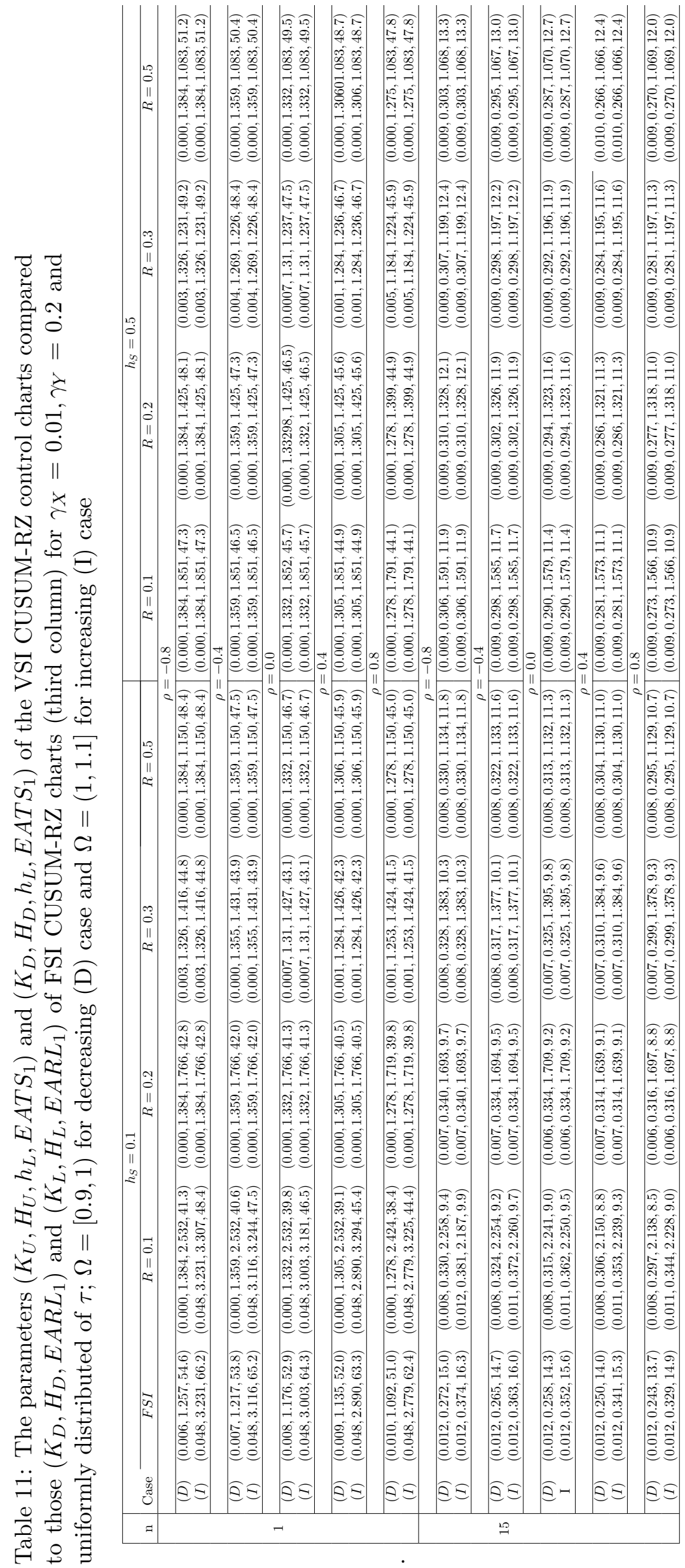




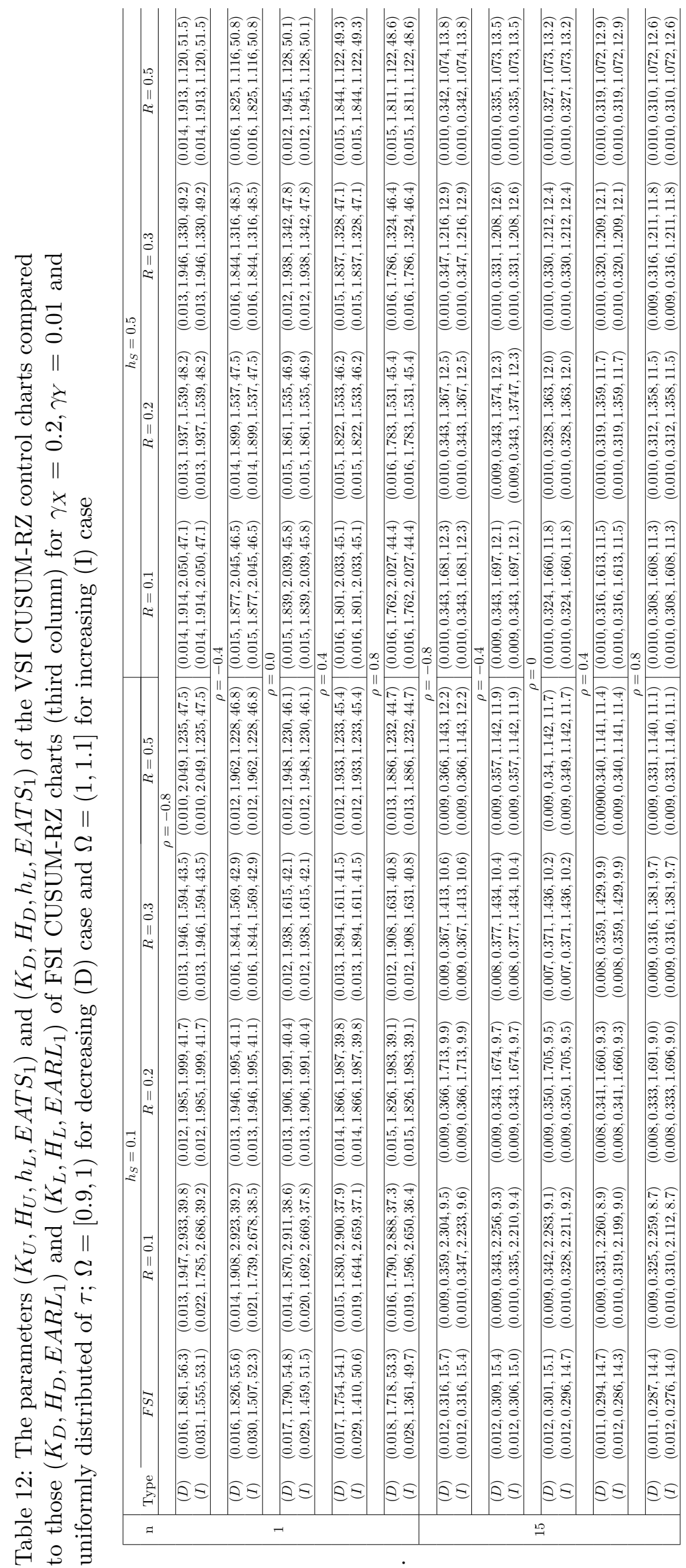




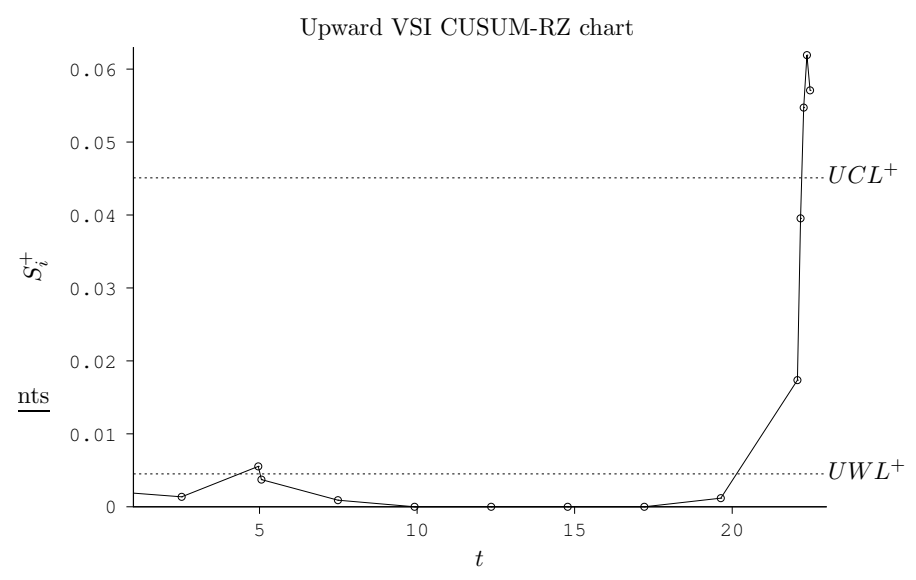

Figure 3: Upward VSI CUSUM-RZ control charts for the food industry example

minutes. The pumpkin seeds and flaxseeds from the muesli mixture in each box are separated by a mechanical procedure and then the sample average weights $\bar{W}_{p, i}=\frac{1}{n} \sum_{j=1}^{n} W_{p, i, j}$ and $\bar{W}_{f, i}=\frac{1}{n} \sum_{j=1}^{n} W_{f, i, j}$ are recorded. Finally, the ratio $\hat{Z}_{i}=\frac{\bar{W}_{p, i}}{\bar{W}_{f, i}}$ is computed and plotted on the upward VSI CUSUM-RZ control chart. As in Celano and Castagliola ${ }^{1}$, we suppose that both $W_{p, i, j}$ and $W_{f, i, j}$ can be well approximated as normal variables with constant CV $\gamma_{p}=0.02$ and $\gamma_{f}=0.01$, i.e. $W_{p, i, j} \sim N\left(\mu_{p, i}, 0.02 \times \mu_{p, i}\right)$ and $W_{f, i, j} \sim N\left(\mu_{f, i}, 0.01 \times \mu_{f, i}\right)$. In addition, the in-control correlation coefficient between these two variables is $\rho_{0}=0.8$. We aslo assume an increase in the ratio and small warning limit coeeficient, that is $\tau=1.01$ and $R_{U}=0.1$. With these parameters, the optimal chart parameters for the upward VSI CUSUM-RZ chart are $h_{S}=0.1, h_{L}=2.43, K_{U}=0.0008191$, and $H_{U}=2.4297865$. From these results, we get, $K^{+}=K_{U} \times z_{0}=0.0008191$, $U C L^{+}=H_{U} \times z_{0}=0.0450865$ and $U W L^{+}=R_{U} \times U C L^{+}=0.00450865$.

Table 13 displays the set of simulated sample data collected from the process, the corresponding box sizes $250-500 \mathrm{gr}$, the sample ratio $\hat{Z}_{i}$, the statistic $S_{i}^{+}$and the total sampling interval. According to the obtained results, the process is assumed to run in-control up to sample \#12. Figure 3 shows the upward VSI CUSUM-RZ control chart, which signals the occurrence of the out-of-control condition by plotting point \#13 above the control limit $U C L^{+}=0.00450865$ (see also bold values in Table 13). The process is allowed to continue, while corrective actions are started by the repair crew to restore the process back to the in-control condition. 


\begin{tabular}{|c|c|c|c|c|c|c|c|c|c|c|c|}
\hline Sample & SI & Total time & Box Size & & & $\begin{array}{l}W_{p, i, j}[\mathrm{gr}] \\
W_{f, i, j}[\mathrm{gr}]\end{array}$ & & & $\begin{array}{l}\bar{W}_{p, i}[\mathrm{gr}] \\
\bar{W}_{f, i}[\mathrm{gr}]\end{array}$ & $\hat{Z}_{i}=\frac{\bar{W}_{p, i}}{\bar{W}_{f, i}}$ & $S_{i}^{+}$ \\
\hline 1 & 0.1 & 0.1 & $\begin{array}{l}250 \mathrm{gr} \\
25.218\end{array}$ & $\begin{array}{l}25.479 \\
25.171\end{array}$ & $\begin{array}{l}25.355 \\
24.684\end{array}$ & $\begin{array}{l}24.027 \\
25.052\end{array}$ & $\begin{array}{l}25.792 \\
25.107\end{array}$ & $\begin{array}{l}24.960 \\
25.046\end{array}$ & 25.122 & 1.003 & 0.002207 \\
\hline 2 & 2.43 & 2.53 & $250 \mathrm{gr}$ & $\begin{array}{l}25.359 \\
25.211\end{array}$ & $\begin{array}{l}25.172 \\
25.115\end{array}$ & $\begin{array}{l}24.508 \\
24.679\end{array}$ & $\begin{array}{l}25.292 \\
24.933\end{array}$ & $\begin{array}{l}24.449 \\
24.831\end{array}$ & $\begin{array}{l}24.956 \\
24.954\end{array}$ & 1.003 & 0.001413 \\
\hline 3 & 2.43 & 4.96 & $250 \mathrm{gr}$ & $\begin{array}{l}24.574 \\
24.784\end{array}$ & $\begin{array}{l}24.864 \\
24.868\end{array}$ & $\begin{array}{l}25.865 \\
25.377\end{array}$ & $\begin{array}{l}25.107 \\
24.879\end{array}$ & $\begin{array}{l}24.811 \\
24.734\end{array}$ & $\begin{array}{l}25.044 \\
24.929\end{array}$ & 1.005 & 0.005620 \\
\hline 4 & 0.1 & 5.06 & $250 \mathrm{gr}$ & $\begin{array}{l}25.313 \\
25.338\end{array}$ & $\begin{array}{l}24.483 \\
24.859\end{array}$ & $\begin{array}{l}24.088 \\
24.305\end{array}$ & $\begin{array}{l}25.184 \\
25.115\end{array}$ & $\begin{array}{l}25.681 \\
25.251\end{array}$ & $\begin{array}{l}24.950 \\
24.974\end{array}$ & 0.999 & 0.003826 \\
\hline 5 & 2.43 & 7.49 & $250 \mathrm{gr}$ & $\begin{array}{l}25.557 \\
25.277\end{array}$ & $\begin{array}{l}24.959 \\
25.402\end{array}$ & $\begin{array}{l}25.023 \\
25.012\end{array}$ & $\begin{array}{l}24.482 \\
24.937\end{array}$ & $\begin{array}{l}25.531 \\
25.148\end{array}$ & $\begin{array}{l}25.111 \\
25.163\end{array}$ & 0.998 & 0.001033 \\
\hline 6 & 2.43 & 9.92 & $250 \mathrm{gr}$ & $\begin{array}{l}24.882 \\
24.962\end{array}$ & $\begin{array}{l}24.473 \\
24.644\end{array}$ & $\begin{array}{l}24.814 \\
24.817\end{array}$ & $\begin{array}{l}25.418 \\
25.419\end{array}$ & $\begin{array}{l}24.732 \\
24.818\end{array}$ & $\begin{array}{l}24.864 \\
24.932\end{array}$ & 0.997 & 0.000000 \\
\hline 7 & 2.43 & 12.35 & $500 \mathrm{gr}$ & $\begin{array}{l}49.848 \\
49.993\end{array}$ & $\begin{array}{l}48.685 \\
49.128\end{array}$ & $\begin{array}{l}49.994 \\
49.830\end{array}$ & $\begin{array}{l}49.910 \\
49.566\end{array}$ & $\begin{array}{l}49.374 \\
49.422\end{array}$ & $\begin{array}{l}49.562 \\
49.588\end{array}$ & 0.999 & 0.000000 \\
\hline 8 & 2.43 & 14.78 & $500 \mathrm{gr}$ & $\begin{array}{l}49.668 \\
49.695\end{array}$ & $\begin{array}{l}50.338 \\
50.681\end{array}$ & $\begin{array}{l}49.149 \\
49.640\end{array}$ & $\begin{array}{l}47.807 \\
48.969\end{array}$ & $\begin{array}{l}49.064 \\
49.612\end{array}$ & $\begin{array}{l}49.205 \\
49.720\end{array}$ & 0.990 & 0.000000 \\
\hline 9 & 2.43 & 17.21 & $500 \mathrm{gr}$ & $\begin{array}{l}51.273 \\
50.366\end{array}$ & $\begin{array}{l}48.303 \\
49.210\end{array}$ & $\begin{array}{l}48.510 \\
49.844\end{array}$ & $\begin{array}{l}50.594 \\
49.890\end{array}$ & $\begin{array}{l}48.591 \\
49.595\end{array}$ & $\begin{array}{l}49.454 \\
49.781\end{array}$ & 0.993 & 0.000000 \\
\hline 10 & 2.43 & 19.64 & $500 \mathrm{gr}$ & $\begin{array}{l}48.720 \\
49.721\end{array}$ & $\begin{array}{l}51.566 \\
50.215\end{array}$ & $\begin{array}{l}49.677 \\
50.178\end{array}$ & $\begin{array}{l}50.651 \\
50.324\end{array}$ & $\begin{array}{l}50.344 \\
50.071\end{array}$ & $\begin{array}{l}50.192 \\
50.102\end{array}$ & 1.002 & 0.001207 \\
\hline 11 & 2.43 & 22.07 & $500 \mathrm{gr}$ & $\begin{array}{l}51.372 \\
50.164\end{array}$ & $\begin{array}{l}51.700 \\
50.272\end{array}$ & $\begin{array}{l}51.000 \\
49.884\end{array}$ & $\begin{array}{l}50.886 \\
50.061\end{array}$ & $\begin{array}{l}49.641 \\
49.845\end{array}$ & $\begin{array}{l}50.920 \\
50.045\end{array}$ & 1.017 & 0.017413 \\
\hline 12 & 0.1 & 22.17 & $500 \mathrm{gr}$ & $\begin{array}{l}52.020 \\
50.749\end{array}$ & $\begin{array}{l}53.182 \\
50.369\end{array}$ & $\begin{array}{l}51.374 \\
49.697\end{array}$ & $\begin{array}{l}51.342 \\
49.575\end{array}$ & $\begin{array}{l}48.771 \\
49.440\end{array}$ & $\begin{array}{l}51.138 \\
49.966\end{array}$ & 1.023 & 0.039620 \\
\hline 13 & 0.1 & 22.27 & $500 \mathrm{gr}$ & $\begin{array}{l}52.360 \\
50.047\end{array}$ & $\begin{array}{l}49.412 \\
49.981\end{array}$ & $\begin{array}{l}50.704 \\
50.297\end{array}$ & $\begin{array}{l}50.370 \\
50.408\end{array}$ & $\begin{array}{l}50.901 \\
50.026\end{array}$ & $\begin{array}{l}50.949 \\
50.152\end{array}$ & 1.016 & 0.054826 \\
\hline 14 & 0.1 & 22.37 & $500 \mathrm{gr}$ & $\begin{array}{l}52.498 \\
50.064\end{array}$ & $\begin{array}{l}50.447 \\
50.124\end{array}$ & $\begin{array}{l}48.713 \\
49.162\end{array}$ & $\begin{array}{l}48.574 \\
48.865\end{array}$ & $\begin{array}{l}50.275 \\
50.344\end{array}$ & $\begin{array}{l}50.101 \\
49.712\end{array}$ & 1.008 & 0.062033 \\
\hline 15 & 0.1 & 22.47 & $250 \mathrm{gr}$ & $\begin{array}{l}25.123 \\
25.041\end{array}$ & $\begin{array}{l}24.658 \\
24.790\end{array}$ & $\begin{array}{l}24.468 \\
24.835\end{array}$ & $\begin{array}{l}25.030 \\
25.211\end{array}$ & $\begin{array}{l}25.071 \\
25.008\end{array}$ & $\begin{array}{l}24.870 \\
24.977\end{array}$ & 0.996 & 0.057239 \\
\hline
\end{tabular}

Table 13: The food industry example data 


\section{Concluding remarks}

We have investigated in this paper a new type of control charts for monitoring the ratio $Z=X / Y$ of two normal variables by incorporating the VSI chart type into CUSUM chart type. The sampling interval of the VSI CUSUM-RZ charts is enabled to vary at different levels corresponding to the previous sample point on the charts. The new coefficient describing the relation between control limit and warning limit is introduced, making the choice of the sampling interval in safe region more reasonable in choosing A direct comparison shows that the VSI CUSUM-RZ charts significantly outperforms the standard CUSUM-RZ charts by producing considerably smaller values of average time to signal $A T S_{1}$ or expected average time to signal $E A T S_{1}$. The results also generally show that the smaller value of $R_{U}$ or $R_{D}$, the better the performance of proposed charts. This leads to an efficient way to improve the chart performance by assigning the value of warning limit coefficients as small as possible.

\section{References}

1. Celano, G., Castagliola, P.. Design of a phase II Control Chart for Monitoring the Ratio of two Normal Variables. Quality and Reliability Engineering International 2016;32(1):291-308.

2. Geary, R.. The Frequency Distribution of the Quotient of Two Normal Variates. Journal of the Royal Statistical Society 1930;93(3):442-446.

3. Marsaglia, G.. Ratios of Normal Variables and Ratios of Sums of Uniform Variables. Journal of the American Statistical Association 1964;60:193-204.

4. Hinkley, D.. On the Ratio of Two Correlated Normal Random Variables. Biometrika 1969;56(3):635-639.

5. Celano, G., Castagliola, P.. A Synthetic Control Chart for Monitoring the Ratio of Two Normal Variables. Quality and Reliability Engineering International 2016;32(2):681-696.

6. Celano, G., Castagliola, P., Faraz, A., Fichera, S.. Statistical Performance of a Control Chart for Individual Observations Monitoring the Ratio of two Normal Variables. Quality and Reliability Engineering International 2014;30(8):1361-1377.

7. Tran, K., Castagliola, P., Celano, G.. Monitoring the Ratio of Two Normal Variables Using Run Rules Type Control Charts. International Journal of Production Research 2016;54(6):1670-1688. 
8. Tran, K., Castagliola, P., Celano, G.. Monitoring the Ratio of Population Means of a Bivariate Normal distribution using CUSUM Type Control Charts. Statistical Papers 2016;In press, DOI: 10.1007/s00362016-0769-4.

9. Tran, K., Castagliola, P., Celano, G.. Monitoring the Ratio of Two Normal Variables Using EWMA Type Control Charts. Quality and Reliability Engineering International 2016;32(2):1853-1869.

10. Tran, K.P., Knoth, S.. Steady-state arl analysis of arl-unbiased ewmarz control chart monitoring the ratio of two normal variables. Qual Reliab Eng Int 2018;:1-14.

11. Castagliola, P., Achouri, A., Taleb, H., Celano, G., Psarakis, S.. Monitoring the coefficient of variation using a variable sampling interval control chart. Quality and Reliability Engineering International 2013;29(8):1135-1149.

12. Saccucci M.S., R.W.A., J.M., L.. Exponentially weighted moving average control schemes with variable sampling intervals. Commun Statist-Simula 1992;21(3):627-657.

13. Brook, D., Evans, D.. An approach to the probability distribution of CUSUM run length. Biometrika 1972;59(3):539-549.

14. Castagliola, P.. A New $S^{2}$-EWMA Control Chart for Monitoring the Process Variance. Quality and Reliability Engineering International 2005;21(8):781-794.

15. W.C.Yeong Michael B.C. Khoo, L.t..M.. Monitoring the coefficient of variation using a variable sampling interval ewma chart. Journal of Quality Technology 2017;49(3):380-401.

16. Tran, P., Tran, K.P.. The efficiency of CUSUM schemes for monitoring the coefficient of variation. Applied Stochastic Models in Business and Industry 2016;32(6):870-881. 